\title{
Usabilidad y satisfacción de una aplicación móvil para el entrena- miento de competencias clínicas
}

\section{Usability and satisfaction of a mobile application for clinical skills training}

\author{
Anabel de la Rosa Gómez ${ }^{1}$ \\ https://orcid.org/0000-0002-3527-1500 \\ Germán Alejandro Miranda Díaz ${ }^{2}$ \\ https://orcid.org/0000-0002-1629-6286 \\ Samanta Xiadani Mendoza Castillo 3 \\ https://orcid.org/0000-0001-7199-8427 \\ Universidad Nacional Autónoma de México
}

Recibido: 06-01-2020

Aceptado: 22-04-2020

\section{Cita Recomendada}

De la Rosa, A., Miranda, G. \& Mendoza, S. (2020).Usabilidad y satisfacción de una aplicación móvil para el entrenamiento de competencias clínicas. Hamut'ay 7(1), 48-59.

http://dx.doi.org/10.21503/hamu.v7i1.1908

\section{RESUMEN}

Los estudiantes de psicología en línea necesitan capacitación profesional que les brinde las habilidades y la eficiencia para enfrentar los problemas de salud emocional. Una de las habilidades más desafiantes es establecer una relación terapéutica funcional con los usuarios. El uso del internet y la tecnología móvil en el campo de la educación dio pauta al surgimiento del m-learning o aprendizaje móvil que posibilita la transportabilidad, conectividad, interactividad y ubicuidad para facilitar el aprendizaje individual o colaborativo al propio ritmo del usuario. No obstante, la constante evolución de las tecnologías exige que los dispositivos móviles tengan atributos más específicos y el crecimiento acelerado ha permitido que se utilicen aplicaciones que no han sido evaluadas para conocer la calidad y funcionamiento desde la perspectiva del usuario meta; de no hacerlo, la tasa de abandono es alta. Es así como el presente estudio tuvo la finalidad de evaluar la usabilidad y satisfacción de una aplicación móvil para el entrenamiento de competencias clínicas (empatía emocional y cognitiva, bienestar psicológico y sentido de presencia) a través de técnicas basadas en mindfulness. Participaron voluntariamente 90 estudiantes de psicología mediante muestreo no probabilistico. El alcance de este estudio fue de tipo exploratorio, se utilizó un diseño pre-experimental post-prueba con un solo grupo. Los estudiantes consideraron que se trata de un entrenamiento novedoso para el desarrollo de sus habilidades clínicas en el que se mostró una media de 6.3 en las puntaciones de usabilidad (muy buena) y una media de 7.4 en satisfacción (buena).

Palabras clave: : aprendizaje móvil, tecnología educativa, competencias clínicas, educación en línea

\footnotetext{
1 Doctora en Psicología. Profesora de carrera, tiempo completo. Responsable académica del Centro de Apoyo Psicológico y Educativo a Distancia (CAPED) y del Laboratorio de Psicología e Innovación Tecnológica (LABPSIIT). anabel.delarosa@iztacala.unam.mx 2 Doctor en Psicología. Profesor de carrera, tiempo completo. Coordinador del Grupo Comunidad de Habilidades y Aprendizaje con Tecnología (CHAT). amiranda@iztacala.unam.mx

3 Miembro del Laboratorio de Psicología e Innovación Tecnológica (LABPSIIT). samanta_mendoza@ired.unam.mx
} 


\section{Abstract}

Online psychology students need professional training that gives them the skills and efficiency to deal with emotional health problems. One of the most challenging skills is to establish a functional therapeutic relationship with users. The use of the internet and mobile technology in the field of education gave rise to mobile learning that allows transportability, connectivity, interactivity and ubiquity to facilitate individual or collaborative learning at the user's own pace. However, the constant evolution of technologies requires that mobile devices have more specific attributes and the accelerated growth has allowed applications that have not been evaluated to be used to know the quality and performance from the perspective of the target user; otherwise, the dropout rate is high. Thus, the present study measured the usability and satisfaction of a mobile application for training clinical skills (emotional and cognitive empathy, psychological well-being and sense of presence) through mindfulness-based techniques. Ninety psychology students voluntarily participated through non-probabilistic sampling. The scope of this study was exploratory, a pre-experimental post-test design with a single group was used. The students considered that it is an innovative training for the development of their clinical skills. The application showed an average of 6.3 in the usability scores (very good) and an average of 7.4 in satisfaction (good).

Keywords: mobile learning, educative technology, clinical skills, e-learning

\section{INTRODUCCIÓN}

La formación de psicólogos en el ámbito clínico es compleja dado que implica no sólo la adquisición de conocimientos y habilidades incluyendo teorías y técnicas de evaluación, diagnóstico e intervención, si no que involucra la percepción simultánea de expresiones verbales y no verbales del paciente-usuario, la autorregulación de sus propias percepciones y emociones, así como el desarrollo de la empatía. No obstante, una de las habilidades más desafiantes y cruciales que deben desarrollar los nuevos terapeutas es cómo establecer una relación terapéutica eficaz, la cual es difícil de medir; sin embargo, se estima que puede representar hasta un 30\% de la varianza del resultado clínico, así como se ha informado que existe una correlación positiva, fuerte y significativa entre la empatía del terapeuta y los resultados terapéuticos $(\mathrm{r}=.82)$ (Hick \& Bien, 2010).

Tradicionalmente, enseñar a los nuevos psicoterapeutas cómo desarrollar una relación terapéutica eficaz se ha centrado en habilidades como la escucha reflexiva o la asistencia. No obstante, algunos autores sugieren que la presencia terapéutica es pre- dictora de una relación terapéutica efectiva (Howgego et al., 2003). Se entiende como presencia a la medida en que se establece una conexión plena con las personas que se atienden y es uno de los factores más importantes para fomentar una respuesta positiva a través del vínculo terapéutico (McDonough-Means, Kreitzer \& Bell, 2004).

Diversos estudios sobre la eficacia de la psicoterapia indican que, con independencia del modelo, método o técnica que se aplique, la relación terapéutica es uno de los factores que más inciden en los resultados positivos (Norcross, Beutler \& Levant, 2005; Siegel, 2012a). Es así que en los últimos años ha existido un creciente interés en el potencial terapéutico de la atención plena o mindfulness (Kabat-Zinn, 2009), debido a que ha mostrado tener un papel esencial en la integración neural en el fomento de la salud (Siegel, 2012b).

Adicionalmente, existe evidencia preliminar que sugiere que los terapeutas pueden potenciar los resultados de la intervención con el paciente después de haber recibido entrenamiento en atención plena (Grepmair et al., 2007). Es así como Kadhdan \& Ciarrochi (2013) mencionan que es deseable que el 
psicólogo clínico o psicoterapeuta posea competencias como la empatía, la compasión, la escucha activa, entre otras, que le apoyen para un buen ejercicio profesional. De ahí la relevancia del entrenamiento en mindfulness para coadyuvar a discriminar emociones propias como un elemento clave en el desarrollo de la empatía, que favorecerá el entendimiento de las emociones de la otredad.

Ahora bien, en el ámbito educativo la incorporación de tecnologías digitales en los procesos de enseñanza y aprendizaje ha crecido de forma exponencial gracias a la incorporación de objetos de aprendizaje, recursos educativos abiertos, y creación de aplicaciones digitales, todas disponibles en la red (Mejía \& López, 2016). Así, el uso del internet y la tecnología móvil en el campo de la educación, a través de sus distintos usos y funciones, dio pauta al surgimiento del m-learning o aprendizaje móvil, con el fin de brindar alternativas que contribuyan a la movilidad, conectividad, ubicuidad y permanencia.

El uso constante de dispositivos móviles utilizados para aprendizaje y educación ha posibilitado el acceso a todo tipo de información, entretenimiento o comunicación en todo momento, sin la necesidad de estar presente o someterse a tiempos estipulados para realizar estas actividades. Por lo que Ramírez, Guillen \& Cifuentes (2016) aseguran que la movilidad que ofrecen las plataformas tecnológicas y la conectividad en tiempo real, se ha facilitado la creación de diversas herramientas que orientan con mayor eficiencia las actividades diarias de los usuarios con dispositivos móviles; mensualmente se lanzan aproximadamente 40.000 Apps, a través de las plataformas de distribución digital iTunes store y Google Play, que para el 2014, contaban con más de 2.5 millones de aplicaciones (Apps) disponibles.

De este modo, existe una demanda muy grande de aplicaciones móviles a nivel mundial que cuentan con una calidad aceptable. En este sentido, la experiencia del usuario, entendida como la respuesta emocional, valoración y satisfacción respecto a la aplicación móvil resultado de su interacción, toma relevancia (Hassan-Montero \& Martín-Fernández, 2005). Entonces, un diseño adecuado centrado en el usuario dependerá de resolver las necesidades de interacción dinámicas básicas del sistema que tenga el usuario, las cuales se componen de 4 principales componentes: usuarios, tareas, productividad, usabilidad (Dumas \& Redish, 1993; Bevan, 2009).

Por lo tanto, al momento de diseñar una aplicación móvil para el aprendizaje se debe comprender quiénes serán los usuarios y qué tarea es la que realizarán, así como tomar en cuenta la usabilidad que incluye la navegación, el diseño de pantalla, la terminología, realimentación, consistencia, modalidad, control del usuario y coincidencia con las tareas del usuario (Lindgaard, 1994; Chanchí, Barragán \& Campo, 2018). Por lo que las pruebas de usabilidad pueden servir para mejorar un producto existente.

En este sentido, el presente estudio exploratorio tuvo la finalidad de medir la usabilidad y satisfacción de una aplicación móvil para el entrenamiento de competencias clínicas (empatía emocional y cognitiva, bienestar psicológico y sentido de presencia) a través de técnicas basadas en mindfulness en estudiantes de psicología en modalidad en línea. Se espera que la aplicación móvil fuera valorada por los usuarios meta como una herramienta adecuada en su funcionamiento, útil y satisfactoria.

\section{Mindfulness como intervención para desarrollo de competencias clínicas.}

En el ámbito de la educación, mindful, alude a no prejuzgar y evitar predisponerse a los eventos (Langer, 2000). No obstante, mindfulness se refiere a la atención consciente, intencionada y neutral a lo que ocurre en el presente (Smalley \& Winston, 2010). La atención plena se describe como la práctica de estar presente con las experiencias inmediatas de nuestras vidas y se cultiva a través de la autorregulación de la experiencia de momento a momento, apuntalado por actitudes de aceptación, curiosidad y no juicio (Shapiro et al., 2006), así como la capacidad de inhibir las evaluaciones secundarias para volver la atención al momento presente cuando se distrae.

De este modo, mindfulness además de emplearse como intervención clínica para diversas problemáticas emocionales en población subclínica también puede favorecer el bienestar y la práctica profesional eficaz de los terapeutas (Hick \& Bien, 2010). Diversos estudios revelan que sintonizar más consigo mismo y con los demás mediante la 
práctica de la atención consciente puede mejorar la sensación de bienestar, así como la actitud hacia los pacientes. $\mathrm{Al}$ respecto, un estudio realizado con médicos de atención primaria mostró que aprender a ser consciente previene el estrés profesional y fomenta una actitud positiva hacia los pacientes porque refuerza la entereza en el reto de cuidar de otras personas (Krasner et al., 2009).

Por su parte, McDonough-Means et al. (2004), informaron que una muestra de estudiantes de psicología que brindaron consejería emocional desarrolló mayor empatía después de una intervención de meditación en comparación con sus compañeros de la lista de espera. El común de los hallazgos es la noción de que la presencia con los demás y consigo mismo fomenta la empatía y la compasión, lo que mejora la salud mental y física del profesional.

Con relación al bienestar, en un estudio realizado por O’Donovan (2007) encontró que los niveles más altos de atención se asociaron con una mayor satisfacción en el trabajo, así como una disminución de burnout entre los profesionales de la salud mental. Asimismo, los terapeutas que han participado en un programa de reducción del estrés basado en mindfulness (MBSR, por sus siglas en inglés) han demostrado disminución en niveles de estrés, ansiedad, afecto negativo y rumia, así como una mayor percepción de empatía, afecto positivo y autocompasión; todo lo anterior en comparación con grupos controles (Shapiro, Carlson \& Astin, 2006).

\section{Aprendizaje móvil}

El aprendizaje móvil es definido como el uso de dispositivos móviles o inalámbricos orientados a fomentar el aprendizaje, como apoyo de la educación en general, y en particular a la educación a distancia (Ngy \& Cumming, 2015), dado que por un lado posibilita el aprendizaje permanente $y$, por otro, es un ambiente digital personalizado de consulta cotidiana que irrumpe los contextos de enseñanza- aprendizaje mediados por las Tecnologías de la Información y la Comunicación (TIC).

Las tecnologías móviles permiten mejorar el acceso, la equidad y la calidad de la educación en todo el mundo con la finalidad de apoyar la meta "Educación para todos" (UNESCO, 2016). Se ha mostrado que el aprendizaje móvil posibilita la transportabilidad, conectividad, interactividad y ubicuidad para facilitar el aprendizaje individual o colaborativo al propio ritmo del usuario (Rico \& Agudo, 2016).

$\mathrm{Al}$ respecto, existe evidencia de que el uso de aplicaciones móviles en diversos niveles educativos potencializa el aprendizaje flexible (Sung, Chang \& Liu, 2016), motiva y satisface a los estudiantes al emplear herramientas novedosas que estimulan la curiosidad, la interacción y la colaboración (Sergio, 2012). No obstante, la constante evolución de las TIC exige que los dispositivos móviles tengan atributos más específicos y el crecimiento acelerado ha permitido que se utilicen aplicaciones que no han sido certificadas por un organismo que garantice la calidad, las cuales, al ser empleadas por los usuarios, al tener una mala experiencia con el uso de la App, la tasa de abandono es alta, lo que lleva a una eliminación y por lo tanto una mala calificación. Por lo que es importante probar las aplicaciones antes de su uso general, tomando en cuenta la funcionalidad, la facilidad, la compatibilidad, el rendimiento, la seguridad y la usabilidad (Alcalde-Alvites \& Alvites-Huamani, 2015; Alonso-Arévalo \& Mirón-Canelo, 2017).

\section{Usabilidad y satisfacción de aplicativos digitales.}

La usabilidad se define como "el grado con el que un producto puede ser usado por usuarios específicos para alcanzar objetivos específicos con efectividad, eficiencia y satisfacción, en un contexto de uso específico" (Enríquez \& Casas, 2013, p.27). Asimismo, se relaciona con los atributos de la aplicación, el contexto de uso conformado por los usuarios, las tareas a realizar, el equipamiento (software o hardware a emplear), así como los diversos ambientes que puedan tener un impacto en el uso del aplicativo (Alvites-Huamaní, 2016).

De acuerdo con Escalona (2013) y Massa \& Pesado (2012) para medir el grado de usabilidad que presenta una aplicación móvil se emplean atributos, entre los que destacan: la facilidad de apren- 
dizaje con la que los objetivos son alcanzados al utilizar la aplicación; la facilidad con la que el usuario memoriza la forma de utilizar la aplicación y con la facilidad con la que la vuelven a emplear; los errores que pueden ser emitidos, así como la solución de los mismos lo más rápido y claro, además de brindar un sistema de recuperación de ese error; también la distribución y los formatos para mostrar los contenidos al usuario; la accesibilidad, en la que se toman en cuenta las limitaciones sensoriales del usuario; la seguridad, centrada en los niveles de riesgo a los que los usuarios tienen que afrontar y así como la disponibilidad de mecanismos de control y protección de la aplicación y los datos que el usuario aloja en ella; otra es la portabilidad, la cual se refiere a la capacidad que tiene la aplicación de ser transferida de un medio a otro; y por último, la del contexto, que se centra en los factores del entorno al hacer uso de la aplicación. Los atributos con los que cuenta una aplicación pueden llegar a ser conceptos abstractos que no pueden ser medidos directamente, por lo que para ser medidos tienen que ser asociadas a métricas.

Al respecto, según la ISO 9241-11 (ISO, 2018), la usabilidad es usada como un calificador para referirse a conocimientos de diseńo, competencias, actividades y atributos de diseño que contribuyen a la funcionalidad y a la calidad. Se destaca que, entre los atributos de la usabilidad, la satisfacción es el más subjetivo, convirtiéndose su estimación en un desafío (Chanchí et al., 2018).

En un estudio realizado por Flett et al., (2018) en el que midieron la usabilidad de dos aplicaciones móviles para el entrenamiento en meditación basadas en la atención plena para la mejora en la salud mental, específicamente síntomas depresivos, ansiedad, estrés, ajuste universitario, florecimiento y resiliencia, se encontró que el uso de la aplicación Mindfulness fue alto durante el período de 10 días (utilizado en 8 de 10 días), pero bajó durante el período de uso extendido de 30 días (menos del $20 \%$ utilizó la aplicación más de 2 veces por semana), por lo que éstos usuarios mostraron mejoras significativas en los síntomas depresivos, el ajuste universitario, la resiliencia y la atención plena en relación con los participantes del grupo control. Se concluyó que la práctica breve de meditación de atención plena móvil puede mejorar algunos aspectos de la salud mental negativa a corto plazo y puede fortalecer la salud mental positiva cuando se usa regularmente.

De este modo, existe una relación muy próxima entre la usabilidad y la satisfacción, siendo ésta última una consecuencia de la facilidad y manejabilidad vinculada a la usabilidad (Serrano \& Cebrián, 2014). De acuerdo con la ISO 9241-11, la satisfacción se refiere a las respuestas físicas, cognitivas y emocionales resultado del uso de un sistema, producto o servicio que satisface las necesidades y expectativas del usuario. Así, la dimensión de satisfacción resulta de la valoración de agrado o desagrado del aplicativo evaluado a partir de la experiencia y percepción del usuario y, sustentada en procesos cognitivos y afectivos personales (Gento \& Vivas, 2003).

En particular, existen estudios que han abordado el reto de evaluar el atributo de la satisfacción del usuario al interacturar con aplicaciones móviles diseñadas para la salud mental (Chanchí et al., 2018; Delgado et al., 2018). Al respecto, Delgado et al., (2018) emplearon un método automatizado para monitorear el comportamiento emocional de un usuario durante una prueba de usabilidad en que la expresión facial del usuario era una variable de seguimiento. Utilizaron una web cam para capturar imágenes de manera continua, para que predijera posibles emociones del usuario en tiempo real, con la finalidad de reducir la subjetividad en la determinación del atributo y convertirse en instrumento de apoyo para la ejecución de pruebas de usuario en un laboratorio de usabilidad.

En esta investigación el atributo satisfacción se ve influenciado por el comportamiento racional y/o emocional del usuario, donde se espera que la ausencia de incomodidad y existencia de actitudes positivas hacia la utilización del producto sea de gran agrado. Por lo que concluyeron que el análisis de la satisfacción a partir de la expresión facial del usuario es uno de los métodos menos intrusivos para la obtención de indicadores emocionales a comparación del uso de sensores biométricos articulados y representa un aporte importante de cara a realizar una estimación más objetiva del atributo satisfacción. 


\section{MATERIALES Y MÉTODOS}

\section{Participantes}

La muestra se integró por 90 estudiantes de la Licenciatura en Psicología en modalidad en línea que voluntariamente aceptaron participar en el estudio. La muestra no probabilística, sujeto-tipo quedó conformada por 71 mujeres y 19 hombres con promedio de edad de 39 años, entre 20 y 71 años. Las medidas sociodemográficas al respecto del semestre que cursaban, lugar de residencia y tipo de sistema operativo del teléfono móvil, se describen en la Tabla 1. Los criterios de inclusión fueron: ser estudiantes de psicología en modalidad en línea y contar con un teléfono móvil con sistema operativo Android o IOS.

Tabla 1.

Datos sociodemográficos de los participantes

\begin{tabular}{|c|c|c|c|}
\hline \multicolumn{2}{|l|}{ Variable } & \multirow{2}{*}{$\begin{array}{l}\mathrm{N} \\
19\end{array}$} & \multirow{2}{*}{$\begin{array}{c}\% \\
21.11 \\
\end{array}$} \\
\hline Sexo & Hombre & & \\
\hline & Mujer & 71 & 78.89 \\
\hline \multirow[t]{5}{*}{ Edad } & $20-30$ & 22 & 24.43 \\
\hline & $31-40$ & 23 & 25.56 \\
\hline & $41-50$ & 33 & 36.69 \\
\hline & $51-60$ & 9 & 9.99 \\
\hline & $61-71$ & 3 & 3.33 \\
\hline \multirow[t]{8}{*}{ Semestre } & 2 & 12 & 13.33 \\
\hline & 3 & 4 & 4.44 \\
\hline & 4 & 9 & 10 \\
\hline & 5 & 6 & 6.67 \\
\hline & 6 & 6 & 6.67 \\
\hline & 7 & 6 & 6.67 \\
\hline & 8 & 8 & 8.89 \\
\hline & 9 & 39 & 43.33 \\
\hline \multirow{11}{*}{$\begin{array}{l}\text { Lugar de } \\
\text { residencia }\end{array}$} & CDMX & 35 & 38.89 \\
\hline & Estado de México & 33 & 36.67 \\
\hline & Guanajuato & 1 & 1.11 \\
\hline & Michoacan & 1 & 1.11 \\
\hline & Baja California & 1 & 1.11 \\
\hline & Guerrero & 1 & 1.11 \\
\hline & Hidalgo & 1 & 1.11 \\
\hline & Oaxaca & 5 & 5.56 \\
\hline & Veracruz & 1 & 1.11 \\
\hline & Puebla & 1 & 1.11 \\
\hline & Queretaro & 8 & 2.22 \\
\hline
\end{tabular}

\begin{tabular}{llrr}
\hline \multicolumn{1}{c}{ Variable } & & $\mathrm{N}$ & $\%$ \\
\hline & Tlaxcala & 7 & 7.78 \\
\cline { 2 - 4 } & EE.UU & 1 & 1.11 \\
\hline Sistema & Android & 83 & 92.22 \\
\cline { 2 - 4 } Operativo & IOS & 7 & 7.78 \\
\hline
\end{tabular}

Fuente: Elaboración propia (2020)

\section{Instrumento}

Cuestionario de usabilidad del sistema informático (Computer System Usability Questionnaire, CSUQ) (Lewis, 1995) adaptado a la población mexicana por Hedlefs et al. (2015); mide las reacciones de desempeño de los participantes a un sitio web con escala likert de 1 a 7 puntos, donde 1 significa “Totalmente en desacuerdo" a 7 "Totalmente de acuerdo", presenta tres factores principales: calidad del sistema: ítems del 1 al 6 , calidad de la información: ítems del 7 al 12 y por último, calidad de la interfaz: ítems del 13 al 16. Cuenta con un alfa de Cronbach de 0.96 .

Cuestionario de satisfacción de la interacción del usuario (Questionnaire for user interaction satisfaction, QUIS) (Chin, Diehl \& HCIL, 1988); mide la satisfacción subjetiva de un usuario respecto a la interfaz persona-computadora con escala likert de 0 a 9 puntos, donde 0 significa "Totalmente malo (horrible)" a 9 "Totalmente estupendo (excelente)", como factores principales se encuentran: medida de la satisfacción general del sistema (satisfacción subjetiva del usuario) y la medida de los factores de interfaz específicos (pantalla, terminología, información del sistema, factores de aprendizaje y capacidades del sistema). Cuenta con un alfa de Cronbach de 0.94

\section{Tipo y Diseño}

El alcance de este estudio fue de tipo exploratorio al tener como propósito determinar el funcionamiento de una aplicación móvil para el desarrollo de habilidades clínicas a través de una aplicación móvil.

Se utilizó un diseño pre-experimental post-prueba con un solo grupo (G X O), debido a que se preten- 
día tener un primer acercamiento al objeto de estudio en un contexto real (Campbell \& Stanley, 1982).

\section{Procedimiento}

Para el desarrollo de la aplicación móvil IztaMind se realizaron distintas fases dirigidas a diseñar una herramienta funcional y de calidad. A continuación, se describen:

I. Investigación. Se revisó la naturaleza del problema al indagar la existencia de otras aplicaciones móviles que abordaran el mismo tema para conocer las necesidades a detalle, y contar con un punto de partida que permitió trazar el plan de trabajo inicial.

II. Diseño de concepto. Se definieron los contenidos teóricos que tendría la aplicación y se eligieron los sistemas operativos, Android y IOS, por ser los de mayor cuota de mercado y posibilidad de edición.

III. Diseño de intervención. Se realizó la diagramación que permitió conceptualizar los segmentos de la aplicación de manera esquematizada.

IV. Diseño visual. Consistió en trasladar el contenido teórico al entorno visual por medio de la realización de video/animaciones a través de una plataforma con la finalidad de ser más atractivos para el usuario. Asimismo, se validaron aspectos generales de la aplicación como color, apariencia, forma de botones estilo y tamaños de fuente.

V. Diseño de prototipo. Consistió en realizar de forma detallada la estructura de la aplicación y la forma en que el usuario interactuaría con ella anexando los contenidos teóricos y el cuestionario de usabilidad.

VI. Programación. En esta fase se codificó la aplicación brindando la funcionalidad y anexando el diseño visual al diseño teórico y de contenidos.

VII. Validación. Finalmente, se realizó una convocatoria difundida en redes sociales institucionales y plataforma educativa en la que se les invitaba a participar de forma voluntaria; los participantes interesados se registraron a través de un formulario de Google forms en el que debían aceptar el consentimiento informado y contestar los cuestionarios preclínicos. Posteriormente, se contactaron vía correo electrónico para proporcionarles el manual de usuario, en donde se detalla la guía de uso y frecuencia, así como el link de descarga de la aplicación móvil. Se pidió a los usuarios utilizar la aplicación durante dos meses, dos horas de uso máximo por cada día. Al finalizar el uso de la aplicación, se les contactó vía correo electrónico para proporcionarles un enlace web que los dirigió a los cuestionarios de usabilidad y satisfacción de la aplicación.

Así, la aplicación móvil <Izta-Mind App>, implementa estrategias de aprendizaje, meditación y autoexploración personal con el fin de diagnosticar y pronosticar las mejoras en la ejecución de habilidades terapéuticas con el usuario, además de una posible detección y mejoramiento en factores de riesgo en la salud del psicoterapeuta. Está basado en código Java Script desarrollado por medio del framework: Apache Cordova para dispositivos móviles utilizando CSS3 y HTML5; el uso de este framework generó una aplicación híbrida, la cual se puede ejecutar en las plataformas de Android y iOS (Figura 1 de la página siguiente).

\section{Confidencialidad y consentimiento informado:}

Todos los participantes aceptaron un consentimiento informado donde se señalaba que se respetarían los derechos a la confidencialidad y privacidad de los datos personales y que sólo se emplearían para fines de investigación.

\section{Resultados}

Los resultados obtenidos en el cuestionario de usabilidad y de satisfacción muestran que la aplicación fue ponderada en ambas categorías como "aceptable" para los participantes. Al respecto de la usabilidad se obtuvo puntuaciones entre 4.29 y 7 con una media de 6.3 (D.E. = 1.02), lo que califica a la aplicación como "muy buena" en su uso. En cuanto a la variable satisfacción se obtuvo una puntuación entre 6 y 9 con una media de 7.4 (D.E. = 1.82), que cualifica a la aplicación como "buena". 


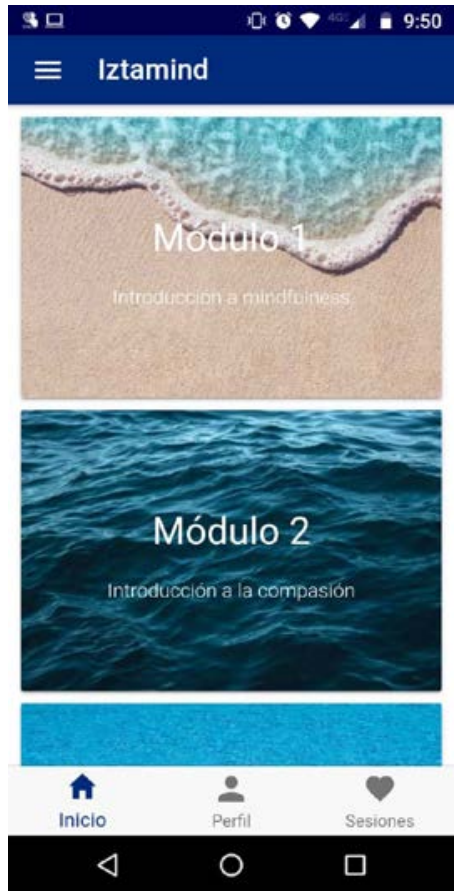

Figura 1.

Aplicación IztaMind.

\section{Medida de usabilidad}

En un análisis específico de las dimensiones de la usabilidad se observó que los usuarios mostraron satisfacción al puntuar entre 4.29 y 6.56 en los reactivos correspondientes a las dimensiones específicas del cuestionario que contienen subcategorías descritas a continuación (Tabla 2).

Al respecto de la calidad del sistema, la satisfacción a la web fue evaluada como satisafactoria [ítems 1 y 4], fácil de uso [ítems 2 y 3] y fácil para aprender [ítems 5 y 6]. Las puntuaciones oscilaron entre 6 y 7 , valorando a la aplicación como "muy buena" en su uso.

En cuanto a la calidad de la información fue evaluada como aceptable; mensajes de error [ítems 7 y 8], mensajes de ayuda [ítems 9 y 11] y organización de la información [ítems 10 y 12]. Las puntuaciones oscilaron entre 4 y 7 , siendo la categoría "mensajes de error" la que mostró puntuación menor.

Asimismo, la calidad de la interfaz fue evaluada como buena; satisfacción de la interfaz [ítems 1 , 14 y 16] y herramientas [ítem 15]. Las puntuaciones oscilaron entre 5 y 7 considerando a la aplicación este último apartado como "buena" en su uso.
Tabla 2.

Medias y desviación estándar por pregunta del cuestionario CSUQ $(\mathrm{n}=90)$

\begin{tabular}{|c|c|c|}
\hline Pregunta & Media & D.E. \\
\hline $\begin{array}{l}\text { 1. Estoy satisfecho con lo fácil que } \\
\text { es utilizar esta App }\end{array}$ & 6.36 & 0.94 \\
\hline 2. Fue simple de usar esta App & 6.52 & 0.77 \\
\hline $\begin{array}{l}\text { 3. Soy capaz de completar mis ta- } \\
\text { reas rápidamente utilizando esta App }\end{array}$ & 6.28 & 0.95 \\
\hline $\begin{array}{l}\text { 4. Me siento cómodo utilizando esta } \\
\text { App }\end{array}$ & 6.46 & 0.89 \\
\hline $\begin{array}{l}\text { 5. Fue fácil aprender a utilizar esta } \\
\text { App }\end{array}$ & 6.56 & 0.81 \\
\hline $\begin{array}{l}\text { 6. Creo que me volví experto rápida- } \\
\text { mente utilizando esta App }\end{array}$ & 6.22 & 0.98 \\
\hline $\begin{array}{l}\text { 7. La App muestra mensajes de } \\
\text { error que me dicen claramente cómo } \\
\text { resolver los problemas }\end{array}$ & 4.29 & 1.92 \\
\hline $\begin{array}{l}\text { 8. Cada vez que cometo un error } \\
\text { utilizando la app, lo resuelvo fácil y } \\
\text { rápidamente }\end{array}$ & 5.76 & 1.4 \\
\hline $\begin{array}{l}\text { 9. La información como ayuda en } \\
\text { línea, mensajes en pantalla y otra } \\
\text { documentación que provee esta App } \\
\text { es clara }\end{array}$ & 5.79 & 1.43 \\
\hline $\begin{array}{l}\text { 10. Es fácil encontrar en la App la } \\
\text { información que necesito }\end{array}$ & 6.08 & 1.09 \\
\hline $\begin{array}{l}\text { 11. La información que proporciona } \\
\text { la App fue efectiva ayudándome a } \\
\text { completar las tareas }\end{array}$ & 6.24 & 0.99 \\
\hline $\begin{array}{l}\text { 12. La organización de la informa- } \\
\text { ción de la información de la App fue } \\
\text { clara }\end{array}$ & 6.4 & 0.78 \\
\hline $\begin{array}{l}\text { 13. La interfaz de la App fue placen- } \\
\text { tera }\end{array}$ & 6.2 & 1.18 \\
\hline 14. Me gustó utilizar la App & 6.42 & 1.06 \\
\hline $\begin{array}{l}\text { 15. La App tuvo todas las herramien- } \\
\text { tas que esperaba que tuviera }\end{array}$ & 5.77 & 1.43 \\
\hline $\begin{array}{l}\text { 16. En general, Estuve satisfecho } \\
\text { con la App }\end{array}$ & 6.27 & 1.03 \\
\hline
\end{tabular}

Nota: D.E. = desviación estandar. Cuestionario de usabilidad del sistema informático (Computer System Usability Questionnaire, CSUQ).

Fuente: elaboración propia (2020).

\section{Medida de satisfacción}

En un análisis específico de las dimensiones de la satisfacción subjetiva del usuario al uso de la app, se observó que los usuarios cualificaron una aplica- 
ción medianamente satisfactoria, al puntuar entre 6.00 y 8.36 los reactivos correspondientes a los factores principales, los que describen en la Tabla 3.

Acerca de la medida de la satisfacción general del sistema, se encontró que la satisfacción subjetiva del usuario fue medianamente aceptable. Los ítems del 1 al 6 mostraron medias entre 7 y 8. Los usuarios consideraron que el uso general del sistema no les causa problema alguno, pero no se encuentra perfecto.

Con relación al nivel de satisfacción sobre los factores de interfaz específicos, se mostró que fueron evaluados como aceptables: (a) pantalla [ítems 7 , 8, 10, 12 y 19]; (b) terminología: [ítems 11,13 y 20]; (c) información del sistema: [ítems 9, 14, 15, 26, 28 y 29]. Las puntuaciones oscilaron entre 6 y 8 , mostrando que la aplicación es aceptable respecto a dichos componentes, aunque desean mayor estructura en la organización de la información.

El nivel de satisfacción sobre los factores de aprendizaje fue cualificado como bastante aceptables [ítems 16, 18 y 24]. Se destaca que esta categoría mostró las puntuaciones más altas en casi todos sus ítems con medias de entre 7 y 9. Los usuarios consideraron que se logra un aprendizaje rápido, que las tareas son sencillas de realizar y los mensajes ayudan para lograr los objetivos.

Finalmente, las capacidades del sistema se ponderaron como aceptables, encontrándose que los ítems 17, 21, 22, 23, 25 y 27 mostraron medias entre 7 y 8 . Los resultados nos muestran que las herramientas que presenta el sistema son básicas y satisfactorias para un uso simple de la aplicación (Tabla 3).

\section{Tabla 3.}

Medias y desviación estándar por pregunta del cuestionario QUIS ( $\mathrm{n}=90)$

\begin{tabular}{lcc}
\hline \multicolumn{1}{c}{ Pregunta } & Media & D.E. \\
\hline 1. Reacciones al software & 7.3 & 1.8 \\
\hline 2. Reacciones al software & 8.0 & 1.6 \\
\hline 3. Reacciones al software & 7.3 & 2.2 \\
\hline 4. Reacciones al software & 7.6 & 1.7 \\
\hline 5. Reacciones al software & 7.2 & 1.8 \\
\hline 6. Reacciones al software & 7.3 & 2.2 \\
\hline 7. Los carácteres de la pantalla son... & 8.3 & 0.9 \\
\hline
\end{tabular}

\begin{tabular}{|c|c|c|}
\hline Pregunta & Media & D.E. \\
\hline $\begin{array}{l}\text { 8. El que la pantalla destaque, } \\
\text { simplifica la tarea }\end{array}$ & 7.9 & 1.4 \\
\hline 9. La organización de la pantalla es... & 7.9 & 1.3 \\
\hline 10. La secuencia de pantalla es... & 7.8 & 1.4 \\
\hline $\begin{array}{l}\text { 11. El uso de términos en todo el } \\
\text { sistema es }\end{array}$ & 7.9 & 1.4 \\
\hline $\begin{array}{l}\text { 12. La posición de los mensajes en } \\
\text { la pantalla es... }\end{array}$ & 7.8 & 1.4 \\
\hline $\begin{array}{l}\text { 13. La terminología es intuitiva con } \\
\text { las tareas que realiza }\end{array}$ & 7.9 & 1.6 \\
\hline $\begin{array}{l}\text { 14. El dispositivo te mantiene infor- } \\
\text { mado sobre lo que esta haciendo }\end{array}$ & 6.6 & 2.3 \\
\hline 15. Los mensajes de error son... & 6.6 & 2.2 \\
\hline $\begin{array}{l}\text { 16. Aprendiendo a manejar el } \\
\text { sistema }\end{array}$ & 8.1 & 1.2 \\
\hline $\begin{array}{l}\text { 17. Explorando nuevas característi- } \\
\text { cas por prueba y error }\end{array}$ & 7.6 & 1.7 \\
\hline $\begin{array}{l}\text { 18.Las tareas pueden ser realizadas } \\
\text { de una manera sencilla }\end{array}$ & 8.3 & 0.8 \\
\hline $\begin{array}{l}\text { 19. Los mensajes de ayuda en la } \\
\text { pantalla son... }\end{array}$ & 7.0 & 2.1 \\
\hline $\begin{array}{l}\text { 20. Los materiales de referencia } \\
\text { suplementarios son... }\end{array}$ & 6.8 & 2.3 \\
\hline 21. La velocidad del sistema es... & 7.6 & 1.6 \\
\hline 22. La confiabilidad del sistema es... & 7.9 & 1.5 \\
\hline 23. El sistema tiende a ser... & 8.2 & 1.1 \\
\hline $\begin{array}{l}\text { 24. Se tienen en cuenta las necesi- } \\
\text { dades de los usuarios con experien- } \\
\text { cia y sin experiencia }\end{array}$ & 7.2 & 2.2 \\
\hline 25. Uso de colores y sonidos & 7.7 & 1.9 \\
\hline $\begin{array}{l}\text { 26. La retroalimentación del sistema } \\
\text { a los errores es... }\end{array}$ & 6.4 & 2.7 \\
\hline $\begin{array}{l}\text { 27. La respuesta del sistema a los } \\
\text { errores es... }\end{array}$ & 6.6 & 2.4 \\
\hline $\begin{array}{l}\text { 28. Los mensajes e informes del } \\
\text { sistema son... }\end{array}$ & 6.5 & 2.4 \\
\hline $\begin{array}{l}\text { 29. El desorden del sistema y el } \\
\text { ruido de la interfaz de usuario son... }\end{array}$ & 6 & 2.8 \\
\hline
\end{tabular}

Nota: D.E. = desviación estandar. Cuestionario de satisfacción de la interacción del usuario (Questionnaire for user interaction satisfaction, QUIS).

Fuente: elaboración propia. 


\section{Discusión y Conclusiones}

El propósito del estudio fue diseñar, desarrollar y medir la usabilidad y satisfacción de uso de una aplicación móvil para el entrenamiento de competencias clínicas (empatía emocional y cognitiva, bienestar psicológico y sentido de presencia) a través de técnicas basadas en mindfulness. Los datos preliminares nos muestran resultados positivos al incorporar tecnologías móviles para el desarrollo de habilidades clínicas en estudiantes universitarios del sistema a distancia y proponer una alternativa prometedora ante la carencia de escenarios supervisados que propicien dichas competencias. En este sentido, nuevas perspectivas para la enseńanza práctica se abren para el desarrollo de tecnología educativa.

Los resultados preliminares obtenidos en la dimensión de usabilidad muestran que la aplicación fue valorada por los estudiantes como muy buena y, en la variable de satisfación, como buena y aceptable; lo cual permitirá, en un siguiente estudio, poner a prueba la eficacia de la aplicación móvil para el desarrollo de competencias clínicas en estudiantes de psicología clínica en formación.

De este modo, los hallazgos coinciden con Serrano \& Cebrián (2014) y Gento \& Vivas (2003) al advertir que el aprendizaje móvil permea con fuerza en los contextos de educación en línea o a distancia como una estrategia que coadyuva para el proceso de enseñanza-aprendizaje, debido a que permite el aprendizaje permanente y posibilita un entorno personalizado y cotidiano. En este sentido, las tecnologías móviles potencian que el aprendizaje se pueda lograr en cualquier momento y lugar; lo cual, propicia un aprendizaje ubicuo (u-learning) al combinar las tecnologías emergentes con los procesos de aprendizaje.

La constante evolución de las TIC exige que los dispositivos móviles tengan atributos más específicos orientados a la educación y a la salud (prevención, diagnóstico, monitoreo). Además, que, al emplear aplicaciones, éstas promuevan una cultura de prevención, viéndose reflejada en la interrelación del trinomio que hay entre el usuario, la tecnología (aplicación móvil) y la salud mental (Mena, Ostos, Félix \& González, 2018). Así, el aplicativo móvil posibilita el aprendizaje en sistemas en línea, presencial y mixto, además de fomentar el autónomo (Pareja, Calle \& Pomposo, 2016).

Como limitaciones del estudio se señala el tamaño de la muestra que podría aumentarse en investigaciones futuras con la finalidad de contar con datos que permitirán la generalización de los resultados a otras muestras con características similares. También, es importante considerar las limitaciones del diseño preexperimental, al no contar con grupo control que permitiera hallazgos más robustos.

\section{Agradecimiento.}

Investigación financiada por el proyecto PAPIME $<$ PE304218>. Consejería emocional. Diseño de una aplicación móvil para el entrenamiento en competencias clínicas en estudiantes a distancia.

\section{REFERENCIAS BIBLIOGRÁFICAS}

AAlcalde-Alvites, M. y Alvites-Huamani, C. (2015). Visión del color y páginas web en educación a distancia. Recuperado de: http://reposital.cuaed.unam.mx:8080/jspui/handle//4594

Alonso-Arévalo, J. y Mirón-Canelo, J. (2017) Aplicaciones móviles en salud: potencial, normativa de seguridad y regulación. Revista Cubana de Información en Ciencias de la Salud, 28(3).

Alvites-Huamaní, P. A. (2016). Usabilidad: Páginas web, entornos y educación virtual. Hamut'ay, 3(1), 71-79. https:// doi.org/10.21503/hamu.v3i1.1002

Bevan, N. (2009). International Standards for Usability Should Be More Widely Used. Journal of Usability Studies, 4 (3), 106-113.

Campbell, D. \& Stanley, J. (1982). Diseños experimentales y cuasiexperimentales en la investigación social. Buenos Aires: Amorrortu Editores.

Chanchí, G. E, Barragán, S. M \& Campo, M. W. Y (2018). Sistema software para el análisis del estrés mental en un test de usuarios. Campus Virtuales, 7 (2), 105-114.

Chin, J. P., Diehl, V. A., \& Norman, K. L. (1988). Development of an instrument measuring user satisfaction of the human-computer interface. In CHI ' 88 Conference Proceedings: Human Factors in Computing Systems, (pp. 213218), New York: Association for Computing Machinery. https://doi.org/10.1145/57167.57203

Delgado A, D. M., Girón T D. F., Chanchí G, G. E., \& 
Márceles V, K. (2018). Propuesta de una herramienta para la estimación de la satisfacción en pruebas de usuario, a partir del análisis de expresión facial. Revista Colombiana de Computación, 19 (2), 6-15.

https://doi.org/10.29375/25392115.3438

Dumas, J.S. \& Redish, J.C. (1993). Una guía práctica para pruebas de usabilidad. Norwood, NJ: Ablex.

Enríquez, J. G. \& Casas, S. I. (2013). Usabilidad en aplicaciones móviles. Universidad Nacional de la Patagonia Austral. Recuperado de http://ict.unpa.edu.ar/files/ICTUNPA-62-2013.pdf

Escalona, Y. (2013). Principios de usabilidad para el diseño de productos de realidad virtual. Avanzada Científica, 1, 4753.

Flett, J. A., Hayne H., Riodan, B. C., Thompson. L. M \& Conner, T. S. (2018). Mobile Mindfulness Meditation: a Randomised Controlled Trial of the Effect of Two Popular Apps on Mental Health. Mindfulness 10, 863-876. https:// doi.org/10.1007/s12671-018-1050-9

Gento, P. S. \& Vivas, G. M. (2003). El SEUE: un instrumento para conocer la satisfacción de los estudiantes universitarios con su educación. Universidad de Los Andes. Acción Pedagógica, 12(2).

Grepmair, L., Mitterlehner, F., Loew, T., Bachler, E., Rother, W. \& Nickel, M. (2007). Promoting mindfulness in psychotherapists in training influences the treatment results of their patients: a randomized, double-blind, controlled study. Psychotherapy and Psychosomatics, 76 (6), 332-338. https:// doi.org/10.1159/000107560

Hassan-Montero, \& Martín-Fernández, F. (2005, 7 de septiembre). La Experiencia del Usuario. NoSoloUsabilidad e-Magazine. Recuperado de http://www.nosolousabilidad. com/articulos/experiencia_del_usuario.htmía 2: 325-333.

Hedlefs, A. M. I., Garza, G. A., Sánchez, M. M. P. \& Garza, V. A. A. (2015). Adaptación al español del Cuestionario de Usabilidad de Sistemas Informáticos CSUQ. Revista Iberoamericana de las Ciencias Computacionales e Informática, 4 (8).

Hick, S. \& Bien, T. (2010). Mindfulness and the Therapeutic Relationship. New York: Guilford Press.

Howgego, I.M., Yellowless, P., Owen, C., Meldrum, L. \& Dark, F. (2003). The therapeutic alliance: the key to effective patient outcome? A descriptive review of the evidence in community mental health case management. The Australian and New Zealand Journal of Psychiatry, 37 (2), 169-83. https://doi.org/10.1046/j.1440-1614.2003.01131.x

ISO. (2018) Ergonomía de la interacción humano-sistema. Parte 11: Usabilidad: definiciones y conceptos. Organización Internacional de Normalización, ISO: ISO/ Plataforma de navegación en línea (OBP). https://www.iso.org/obp/ ui/\#iso:std:iso:9241:-11:ed-2:v1:en

Kabat-Zinn. (2009). Full catastrophe living: Using the wisdom of your body and mind to face stress, pain, and illness.

\section{New York: Bantam Dell.}

Kadhdan, T. \& Ciarrochi, J. (2013). Mindfulness aceptación y psicología positiva: las siete bases del bienestar. Espańa: Ediciones Obelisco.

Krasner, M.S., Epstein, R.M., Beckman, H., et al. (2009). Association of an Educational Program in Mindful Communication with Burnout, Empathy, and Attitudes Among Primary Care Physicians. JAMA, 302 (12),1284-1293. https:// doi.org/10.1001/jama.2009.1384

Langer, E.J., \& Moldoveanu, M.C. (2000). The Construct of Mindfulness. Hillsdale, E. J. NJ Erlbaum. https://doi. org/10.1111/0022-4537.00148

Lewis, J. R. (1995). IBM Computer Usability Satisfaction Questionnaires: Psychometric Evaluation and Instructions for Use. International Journal of Human-Computer Interaction, 7(1), 57-78. https://doi.org/10.1080/10447319509526110

Lindgaard, G. (1994). Pruebas de usabilidad y evaluación del sistema. Londres: Chapman \& Hall.

Massa, S. \& Pesado, P. (2012). Evaluación de la usabilidad de un Objeto de Aprendizaje por estudiantes. Revista Iberoamericana de Educación y Tecnología \& Tecnología en Educación, 8, 65-76. Recuperado de http://sedici.unlp.edu. ar/handle/10915/25537

McDonough-Means, S. \& Kreitzer, M. J. \& Bell, I. R. (2004). Fostering a healing presence and investigating its mediators. Journal of alternative and complementary medicine, 10 (1), 25-41.https://doi.org/10.1089/acm.2004.10.S-25

Mejía, J. \& López, D. (2016). Modelo de Calidad de E-learning para Instituciones de Educación Superior en Colombia, Formación Universitaria, 9(2), 59-72. https://doi. org/10.4067/S0718-50062016000200007

Mena, L., Félix, V., Ochoa, A. Ostos, R., González, E., Aspuru, J., Velarde, P. \& Maestre, G. (2018). Mobile Personal Health Monitoring for Automated Classification of Electrocardiogram Signals in Elderly. Computational and Mathematical Methods in Medicine. https:/doi. org/10.1155/2018/9128054

Ngy, W. \& Cumming, T. M. (2015). Sustaining Mobile Learning: Theory, Research and Practice. UK: Routledge. https://doi.org/10.4324/9781315766553-5

Norcross, J.C., Beutler, L. \& Levant, R. F. (2005). Evidence-Based Practices in Mental Health: Debate and Dialogue on the Fundamental Questions. Washington, DC: American Psychological Association. https://doi.org/10.1037/11265000

O’Donovan, M.O. (2007). Implementing reflection: insights from pre-registration mental health students. Nurse Education Today, 27(6), 10-16. https://doi.org/10.1016/j. nedt.2006.09.001

Pareja, A., Calle, C., \& Pomposo, L. (2016). Aprendiendo a hacer presentaciones efectivas en inglés con BusinessApp. Revista Iberoamericana de Educación a Distancia, 19 (1), 41-61. 
https://doi.org/10.5944/ried.19.1.14616

Ramírez, L., Guillen, E. \& Cifuentes, Y. (2016) Estrategia de validación para aplicaciones móviles de salud. Actas de Ingeniería 2, 325-333.

Rico, M. M., \& Agudo, J. E. (2016). Aprendizaje móvil de inglés mediante juegos de espías en Educación Secundaria. Revista Iberoamericana de Educación a Distancia, 19 (1), 121-139.

Sergio, F. (2012). 10 ways that mobile learning will revolutionize education. Recuperado de www.fastcodesign. com/1669896/10-ways-hat-mobilelearning-will-revolutionize-education

Serrano, A. J \& Cebrián R. D. (2014). Usabilidad y satisfacción de la e-rúbrica. REDU. Revista de Docencia Universitaria, 12 (1), 177-195. https://doi.org/10.4995/ redu. 2014.6426

Shapiro, S.L., Carlson, L.E., Astin, J.A., \& Freedman, B.S. (2006). Mechanisms of mindfulness. Journal of clinical psychology, 62 (3), 373-86.

https://doi.org/10.1002/jclp.20237

Siegel, D. J. (2012a). Mindfulness y Psicoterapia: Técnicas, prácticas de atención plena para psicoterapeutas. Paidos. Barcelona, Buenos Aires, México.

Siegel, D. J. (2012b). The developing mind: How relationships and the brain interact to shape who we are (2nd ed.). New York, NY, US: Guilford Press.

Smalley, S. L., \& Winston, D. (2010). Fully Present: The Science, Art, and Practice of Mindfulness. New York: Da Capo Lifelong Books.

Sung, Y., Chang, K. \& Liu, T. (2016). The effects of integrating mobile devices with teaching and learning on students' learning performance: A meta-analysis and research synthesis. Computers \& Education, 94, 252-275.

https://doi.org/10.1016/j.compedu.2015.11.008

UNESCO. (2016). Informe de Seguimiento de la Educación en el Mundo, 2016: La Educación al servicio de los pueblos y el planeta: creación de futuros sostenibles para todos. 